\title{
Instantly Propagating States
}

\author{
Alexander Soiguine \\ SOiGUINE Quantum Computing, Aliso Viejo, USA \\ Email: alex@soiguine.com
}

How to cite this paper: Soiguine, A. (2021) Instantly Propagating States. Journal of Applied Mathematics and Physics, 9, 468-475.

https://doi.org/10.4236/jamp.2021.93032

Received: January 6, 2021

Accepted: March 22, 2021

Published: March 25, 2021

Copyright (c) 2021 by author(s) and Scientific Research Publishing Inc. This work is licensed under the Creative Commons Attribution International License (CC BY 4.0).

http://creativecommons.org/licenses/by/4.0/

\begin{abstract}
The current work considers the sprefield wave functions received as special g-qubit solutions of Maxwell equations in the terms of geometric algebra. I will call such g-qubits spreons or sprefields. The purpose of this article is to analyze behavior of such wave functions in scattering and measurements. It is shown that sprefields are defined through the whole three-dimensional space at all values of the time parameter. They instantly change all their values when get scattered, that is subjected to Clifford translation. In "measurements", when a sprefield acts on a static geometric algebra element through the Hopf fibration, sprefield collapses and new geometric algebra non static, rotating element is thereby created.
\end{abstract}

\section{Keywords}

Wave Functions, Geometric Algebra, Measurements, Scattering,

Entanglement

\section{Introduction}

Usage of even subalgebra $G_{3}^{+}$of geometric algebra $G_{3}$ [1] [2] [3] stems from generalization of complex numbers [3] [4]. The sprefield wave functions (states) received as special $G_{3}^{+}$solutions of Maxwell equations [5] [6].

In terms of geometric algebra $G_{3}^{+}$, the electromagnetic Maxwell equation in free space

$$
\left(\partial_{t}+\nabla\right) F=0,
$$

where $F=F_{0} \exp \left[I_{S}(\omega t-k \cdot r)\right]$, has two linear independent solutions [3] [5] [6]:

$$
\left\{\begin{aligned}
F_{+} & =\left(e_{0}+I_{3} h_{0}\right) \exp \left[I_{S} \omega\left(t-\left(I_{3} I_{S}\right) \cdot r\right)\right] \\
& =\left(e_{0}+I_{3} h_{0}\right) \exp \left[I_{S} \omega t\right] \exp \left[-I_{S}\left[\left(I_{3} I_{S}\right) \cdot r\right]\right] \\
F_{-} & =\left(e_{0}+I_{3} h_{0}\right) \exp \left[I_{S} \omega\left(t+\left(I_{3} I_{S}\right) \cdot r\right)\right] \\
& =\left(e_{0}+I_{3} h_{0}\right) \exp \left[I_{S} \omega t\right] \exp \left[I_{S}\left[\left(I_{3} I_{S}\right) \cdot r\right]\right]
\end{aligned}\right.
$$


For arbitrary scalars $\lambda$ and $\mu$ :

$$
\lambda F_{+}+\mu F_{-}=\left(e_{0}+I_{3} h_{0}\right) \mathrm{e}^{I_{S} \omega t}\left(\lambda \mathrm{e}^{-I_{S}\left[\left(I_{3} I_{S}\right) \cdot r\right]}+\mu \mathrm{e}^{I_{S}\left[\left(I_{3} I_{S}\right) \cdot r\right]}\right)
$$

is also solution of (1.1). The item in the second parenthesis is weighted linear combination of two states with the same phase in the same plane but opposite sense of orientation. These states are strictly coupled because bivector plane should be the same for both, does not matter what happens with that plane.

Formula (1.3) does not immediately look like an element of $G_{3}^{+}$due to the factor $\left(e_{0}+I_{3} h_{n}\right)$. But necessary transformations of the initial bivector basis $\left\{B_{1}, B_{2}, B_{3}\right\}$ into triple of unit value orthonormal bivectors $\left\{I_{S}, I_{B_{0}}, I_{E_{0}}\right\}$ where $I_{S}$ is unit value bivector, dualto the propagation direction vectork; unit value $I_{B_{0}}$ is dual to initial vector of magnetic field $h_{0}$; unit value $I_{E_{0}}$ is dual to initial vector of electric field $e_{0}$, change (1.3) into:

$$
\lambda \mathrm{e}^{I_{\text {Plane }}^{+} \varphi^{+}}+\mu \mathrm{e}^{I_{\text {Plane }}^{-} \varphi^{-}}
$$

where

$$
\begin{gathered}
\varphi^{ \pm}=\cos ^{-1}\left(\frac{1}{\sqrt{2}} \cos \omega\left(t \mp\left[\left(I_{3} I_{S}\right) \cdot r\right]\right)\right), \\
I_{\text {Plane }}^{ \pm}=I_{S} \frac{\sin \omega\left(t \mp\left[\left(I_{3} I_{S}\right) \cdot r\right]\right)}{\sqrt{1+\sin ^{2} \omega\left(t \mp\left[\left(I_{3} I_{S}\right) \cdot r\right]\right)}}+I_{B_{0}} \frac{\cos \omega\left(t \mp\left[\left(I_{3} I_{S}\right) \cdot r\right]\right)}{\sqrt{1+\sin ^{2} \omega\left(t \mp\left[\left(I_{3} I_{S}\right) \cdot r\right]\right)}} \\
+I_{E_{0}} \frac{\sin \omega\left(t \mp\left[\left(I_{3} I_{S}\right) \cdot r\right]\right)}{\sqrt{1+\sin ^{2} \omega\left(t \mp\left[\left(I_{3} I_{S}\right) \cdot r\right]\right)}}
\end{gathered}
$$

The expression (1.4) is linear combination of two geometric algebra states, g-qubits, which are elements of the form $\alpha+I_{\text {Plane }} \beta$ with some arbitrary unit value bivector $I_{\text {Plane }}$ in three dimensions.

Let us calculate $\lambda \mathrm{e}^{I_{\text {Plane }}^{+} \varphi^{+}}+\mu \mathrm{e}^{I_{\text {Plane }}^{-} \varphi^{-}}$with $\lambda=\mu=1$ (the $\sqrt{2}$ is included for normalization to further write the expression as exponent):

$$
\begin{aligned}
\mathrm{e}^{I_{\text {Plane }}^{+} \varphi^{+}}+\mathrm{e}^{I_{\text {Plane }}^{-} \varphi^{-}} \\
=\frac{1}{\sqrt{2}} \cos \omega\left(t-\left[\left(I_{3} I_{S}\right) \cdot \vec{r}\right]\right)+\frac{1}{\sqrt{2}} I_{S} \sin \omega\left(t-\left[\left(I_{3} I_{S}\right) \cdot \vec{r}\right]\right) \\
+\frac{1}{\sqrt{2}} I_{B_{0}} \cos \omega\left(t-\left[\left(I_{3} I_{S}\right) \cdot \vec{r}\right]\right)+\frac{1}{\sqrt{2}} I_{E_{0}} \sin \omega\left(t-\left[\left(I_{3} I_{S}\right) \cdot \vec{r}\right]\right) \\
+\frac{1}{\sqrt{2}} \cos \omega\left(t+\left[\left(I_{3} I_{S}\right) \cdot \vec{r}\right]\right)+\frac{1}{\sqrt{2}} I_{S} \sin \omega\left(t+\left[\left(I_{3} I_{S}\right) \cdot \vec{r}\right]\right) \\
+\frac{1}{\sqrt{2}} I_{B_{0}} \cos \omega\left(t+\left[\left(I_{3} I_{S}\right) \cdot \vec{r}\right]\right)+\frac{1}{\sqrt{2}} I_{E_{0}} \sin \omega\left(t+\left[\left(I_{3} I_{S}\right) \cdot \vec{r}\right]\right) \\
=\frac{2}{\sqrt{2}} \cos \omega\left(\left[\left(I_{3} I_{S}\right) \cdot \vec{r}\right]\right)\left(\cos \omega t+I_{S} \sin \omega t+I_{B_{0}} \cos \omega t+I_{E_{0}} \sin \omega t\right)
\end{aligned}
$$

I will call such g-qubits spreons or sprefields. The purpose of this article is to analyze behavior of such wave functions in scattering and measurements. 


\section{Scattering of Sprefields}

The sprefield wave function:

$$
\begin{aligned}
& S p\left(\omega, \vec{r}, t, B_{1}, B_{2}, B_{3}\right) \\
& =\sqrt{2} \cos \omega\left(\left[\left(I_{3} B_{1}\right) \cdot \vec{r}\right]\right)\left(\cos \omega t+B_{1} \sin \omega t+B_{2} \cos \omega t+B_{3} \sin \omega t\right)
\end{aligned}
$$

can be written, following multiplication rules of basis bivectors, as:

$$
\operatorname{Sp}\left(\omega, \vec{r}, t, B_{1}, B_{2}\right)=R\left(\omega, \vec{r}, B_{1}\right)\left(\mathrm{e}^{B_{1} \omega t}+\mathrm{e}^{-B_{1} \omega t} B_{2}\right)
$$

where $R\left(\omega, \vec{r}, B_{1}\right)$ is a scalar valued function.

The specifics of (2.1)-(2.2) is that it is a non-local field object instantly spreading its modifications, caused by Clifford translations or by Hopf fibrations ("measurements"), through the whole $3 \mathrm{D}$ and time parameter values. In the Hopf fibration new element is created, not static one, opposite to the measured $G_{3}^{+}$element, with stable rotation characteristics depending on the sprefield wave function parameters.

The scheme suggested in the current text is based on manipulation and transferring of quantum states (wave functions) as operators acting on observables, both formulated in terms of geometrical algebra. Wave functionsact in the current context on static $G_{3}^{+}$elements through measurements, creating "particles".

Normalized wave functions as elements of $G_{3}^{+}$are naturally mapped onto unit sphere $\mathbb{S}^{3}$. Two-state system is then just a couple of points on $\mathbb{S}^{3}$, $\left\{\alpha_{1}, \beta_{1} b_{1}^{1}, \beta_{1} b_{1}^{2}, \beta_{1} b_{1}^{3}\right\}$ and $\left\{\alpha_{2}, \beta_{2} b_{2}^{1}, \beta_{2} b_{2}^{2}, \beta_{2} b_{2}^{3}\right\}$, corresponding to wave functions:

$$
\begin{gathered}
\mathrm{e}^{I_{S_{1}} \varphi_{1}}=\alpha_{1}+I_{S_{1}} \beta_{1}=\alpha_{1}+\beta_{1} b_{1}^{1} B_{1}+\beta_{1} b_{1}^{2} B_{2}+\beta_{1} b_{1}^{3} B_{3} \\
\mathrm{e}^{I_{S_{2}} \varphi_{2}}=\alpha_{2}+I_{S_{2}} \beta_{2}=\alpha_{2}+\beta_{2} b_{2}^{1} B_{1}+\beta_{2} b_{2}^{2} B_{2}+\beta_{2} b_{2}^{3} B_{3}
\end{gathered}
$$

with

$$
\begin{aligned}
& \left(\alpha_{1}\right)^{2}+\left(\beta_{1}\right)^{2}\left(\left(b_{1}^{1}\right)^{2}+\left(b_{1}^{2}\right)^{2}+\left(b_{1}^{3}\right)^{2}\right)=\left(\alpha_{1}\right)^{2}+\left(\beta_{1}\right)^{2}=1 \\
& \left(\alpha_{2}\right)^{2}+\left(\beta_{2}\right)^{2}\left(\left(b_{2}^{1}\right)^{2}+\left(b_{2}^{2}\right)^{2}+\left(b_{2}^{3}\right)^{2}\right)=\left(\alpha_{2}\right)^{2}+\left(\beta_{2}\right)^{2}=1
\end{aligned}
$$

in some bivector basis $B_{1} B_{2} B_{3}=1$, with multiplication rules $B_{1} B_{2}=-B_{3}$, $B_{1} B_{3}=B_{2}, \quad B_{2} B_{3}=-B_{1}$.

Then it follows that two wave functions of an arbitrary two-function system are, in any case, connected by the Clifford translation ${ }^{1}$ :

$$
\mathrm{e}^{I_{S_{2}} \varphi_{2}}=\left(\mathrm{e}^{I_{S_{2}} \varphi_{2}} \mathrm{e}^{-I_{S_{1}} \varphi_{1}}\right) \mathrm{e}^{I_{S_{1}} \varphi_{1}} \equiv C l\left(S_{2}, \varphi_{2}, S_{1}, \varphi_{1}\right) \mathrm{e}^{I_{S_{1}} \varphi_{1}}
$$

The product of exponents $\mathrm{e}^{I_{S_{2}} \varphi_{2}} \mathrm{e}^{-I_{S_{1}} \varphi_{1}}$ is trivial in the case $S_{1}=S_{2} \equiv S$ (the case of geometrically unspecified imaginary unit plane in conventional quantum mechanics) $\mathrm{e}^{I_{S_{2}} \varphi_{2}} \mathrm{e}^{-I_{S_{1}} \varphi_{1}}=\mathrm{e}^{I_{S}\left(\varphi_{2}-\varphi_{1}\right)}$. Though in general case we have more complicated result:

${ }^{1}$ It is universally possible due to the hedgehog (hairy ball) theorem which says that there exists nonvanishing continuous tangent vector field on odd-dimensional sphere $\mathbb{S}^{3}$. 


$$
\begin{aligned}
& C l\left(S_{2}, \varphi_{2}, S_{1}, \varphi_{1}\right) \equiv \mathrm{e}^{I_{S_{2}} \varphi_{2}} \mathrm{e}^{-I_{S_{1}} \varphi_{1}} \\
& =\cos \left(\varphi_{1}\right) \cos \left(\varphi_{2}\right)+\left(s_{1} \cdot S_{2}\right) \sin \left(\varphi_{1}\right) \sin \left(\varphi_{2}\right)+I_{3} S_{2} \cos \left(\varphi_{1}\right) \sin \left(\varphi_{2}\right) \\
& \quad+I_{3} S_{1} \cos \left(\varphi_{2}\right) \sin \left(\varphi_{1}\right)+I_{3}\left(s_{2} \times s_{1}\right) \sin \left(\varphi_{1}\right) \sin \left(\varphi_{2}\right)
\end{aligned}
$$

where $s_{1}$ and $s_{2}$ are vectors dual to planes $S_{1}$ and $S_{2}$ matching orientation of $I_{3}$.

The result of Clifford translation (2.4) is a $G_{3}^{+}$element. From knowing Clifford translation connecting any two wave functions as points on $\mathbb{S}^{3}$ it follows that the result of measurement of any observable $C$ by wave function $e^{I_{S_{1}} \varphi_{1}}$, for example $\mathrm{e}^{I_{S_{1}} \varphi_{1}} \mathrm{Ce}^{-I_{S_{1}} \varphi_{1}} \equiv C\left(S_{1}, \varphi_{1}\right)$, immediately gives the result of (not made) measurement by $\mathrm{e}^{I_{S_{2}} \varphi_{2}}$ :

$$
\begin{aligned}
\mathrm{e}^{I_{S_{2}} \varphi_{2}} C \mathrm{e}^{-I_{S_{2}} \varphi_{2}} & =\mathrm{e}^{I_{S_{2}} \varphi_{2}} \mathrm{e}^{-I_{S_{1}} \varphi_{1}} \mathrm{e}^{I_{S_{1}} \varphi_{1}} C \mathrm{e}^{-I_{S_{1}} \varphi_{1}} \mathrm{e}^{I_{S_{1}} \varphi_{1}} \mathrm{e}^{-I_{S_{2}} \varphi_{2}} \\
& =\mathrm{e}^{I_{S_{2}} \varphi_{2}} \mathrm{e}^{-I_{S_{1}} \varphi_{1}} C\left(S_{1}, \varphi_{1}\right) \mathrm{e}^{I_{S_{1}} \varphi_{1}} \mathrm{e}^{-I_{S_{2}} \varphi_{2}} \\
& =C l\left(S_{2}, \varphi_{2}, S_{1}, \varphi_{1}\right) C\left(S_{1}, \varphi_{1}\right) \overline{C l\left(S_{2}, \varphi_{2}, S_{1}, \varphi_{1}\right)^{2}}
\end{aligned}
$$

This is geometrically clear and unambiguous explanation of strict connectivity of the results of measurements instead of "entanglement" in conventional quantum mechanics.

Take the spreon (1.5):

$$
\begin{aligned}
& \operatorname{Sp}\left(\omega, \vec{r}, t, I_{S_{0}}, I_{B_{0}}, I_{E_{0}}\right) \\
& =2 \cos \omega\left(\left[\left(I_{3} I_{S}\right) \cdot \vec{r}\right]\right)\left(\frac{1}{\sqrt{2}} \cos \omega t+\frac{1}{\sqrt{2}} I_{S} \sin \omega t\right. \\
& \left.+\frac{1}{\sqrt{2}} I_{B_{0}} \cos \omega t+\frac{1}{\sqrt{2}} I_{E_{0}} \sin \omega t\right)
\end{aligned}
$$

By redifining for reading formulas easier $I_{S} \equiv B_{1}, \quad I_{B_{0}} \equiv B_{2}, \quad I_{E_{0}} \equiv B_{3}$ we have the following:

Sprefield when scattered by a $G_{3}^{+}$element $\cos \gamma+\sin \gamma\left(\gamma_{1} B_{1}+\gamma_{2} B_{2}+\gamma_{3} B_{3}\right)=\mathrm{e}^{B \gamma}, \quad \gamma_{1} B_{1}+\gamma_{2} B_{2}+\gamma_{3} B_{3} \equiv B$, unit value bivector if $\gamma_{1}^{2}+\gamma_{2}^{2}+\gamma_{3}^{2}=1$, becomes:

$$
\begin{aligned}
\mathrm{e}^{B \gamma} S p\left(\omega, \vec{r}, t, B_{1}, B_{2}\right) & =\mathrm{e}^{B \gamma} R\left(\omega, \vec{r}, B_{1}\right)\left(\mathrm{e}^{B_{1} \omega t}+\mathrm{e}^{-B_{1} \omega t} B_{2}\right) \\
& =R\left(\omega, \vec{r}, B_{1}\right)\left(\mathrm{e}^{B \gamma} \mathrm{e}^{B_{1} \omega t}+\mathrm{e}^{B \gamma} \mathrm{e}^{-B_{1} \omega t} B_{2}\right)
\end{aligned}
$$

Let us use again a general formula for the product of two geometric algebra exponents:

$$
\begin{aligned}
\mathrm{e}^{I_{S_{1}} \alpha} \mathrm{e}^{I_{S_{2}} \beta}= & \cos \alpha \cos \beta-\left(s_{1} \cdot s_{2}\right) \sin \alpha \sin \beta+I_{3} s_{1} \cos \beta \sin \alpha \\
& +I_{3} s_{2} \cos \alpha \sin \beta-I_{3}\left(s_{1} \times s_{2}\right) \sin \alpha \sin \beta
\end{aligned}
$$

where $s_{1}$ and $s_{2}$ are vectors dual correspondingly to bivectors $I_{S_{1}}$ and $I_{S_{2}}$. In $\mathrm{e}^{B \gamma} \mathrm{e}^{B_{1} \omega t}$ we have $\cos \alpha=\cos \gamma, s_{1}=\left(\gamma_{1}, \gamma_{2}, \gamma_{3}\right), \cos \beta=\cos \omega t$, $s_{2}=(1,0,0)$ and in $\mathrm{e}^{B \gamma} \mathrm{e}^{-B_{1} \omega t}, s_{2}=(-1,0,0)$. Thus,

${ }^{2}$ Difference in exponent signs from usual measurement definition is made just for some convenience. It means that the angle has opposite sign or can be thought that the bivector plane was flipped. 


$$
\begin{aligned}
\mathrm{e}^{B \gamma} \mathrm{e}^{B_{1} \omega t}= & \cos \gamma \cos \omega t-\gamma_{1} \sin \gamma \sin \omega t+\left(\gamma_{1} B_{1}+\gamma_{2} B_{2}+\gamma_{3} B_{3}\right) \sin \gamma \cos \omega t \\
& +B_{1} \cos \gamma \sin \omega t+\left(\gamma_{3} B_{2}-\gamma_{2} B_{3}\right) \sin \gamma \sin \omega t \\
\mathrm{e}^{B \gamma} \mathrm{e}^{-B_{1} \omega t}= & \cos \gamma \cos \omega t-\gamma_{1} \sin \gamma \sin \omega t+\left(\gamma_{1} B_{1}+\gamma_{2} B_{2}+\gamma_{3} B_{3}\right) \sin \gamma \cos \omega t \\
& -B_{1} \cos \gamma \sin \omega t+\left(-\gamma_{3} B_{2}+\gamma_{2} B_{3}\right) \sin \gamma \sin \omega t \\
\mathrm{e}^{B \gamma} \mathrm{e}^{-B_{1} \omega t} B_{2}= & B_{2} \cos \gamma \cos \omega t-B_{2} \gamma_{1} \sin \gamma \sin \omega t+\left(-\gamma_{1} B_{3}-\gamma_{2}+\gamma_{3} B_{1}\right) \sin \gamma \cos \omega t \\
+ & B_{3} \cos \gamma \sin \omega t+\left(\gamma_{3}+\gamma_{2} B_{1}\right) \sin \gamma \sin \omega t
\end{aligned}
$$

Then it follows that the result of scattering is:

$$
\begin{aligned}
& R\left(\omega, \vec{r}, B_{1}\right)\left[\cos \gamma \cos \omega t-\gamma_{2} \sin \gamma \cos \omega t+\left(\gamma_{3}-\gamma_{1}\right) \sin \gamma \sin \omega t\right. \\
& +\left(\left(\gamma_{3}+\gamma_{1}\right) \sin \gamma \cos \omega t+\gamma_{2} \sin \gamma \sin \omega t+\cos \gamma \sin \omega t\right) B_{1} \\
& +\left(\gamma_{2} \sin \gamma \cos \omega t+\left(\gamma_{3}-\gamma_{1}\right) \sin \gamma \sin \omega t+\cos \gamma \cos \omega t\right) B_{2} \\
& \left.+\left(\left(\gamma_{3}-\gamma_{1}\right) \sin \gamma \cos \omega t-\gamma_{2} \sin \gamma \sin \omega t+\cos \gamma \sin \omega t\right) B_{3}\right]
\end{aligned}
$$

This scattered sprefield is defined in all points $\vec{r}$ of three-dimensional space and time parameter values $t$ and is obviously independent of when scattering took place.

In some special cases of the scattering element, we get the following:

If sprefield is scattered by $\cos \gamma+\sin \gamma B_{1}$ the result is:

$$
R\left(\omega, \vec{r}, B_{1}\right)\left[\cos (\omega t+\gamma)+\sin (\omega t+\gamma) B_{1}+\cos (\omega t+\gamma) B_{2}+\sin (\omega t-\gamma) B_{3}\right]
$$

If sprefield is scattered by $\cos \gamma+\sin \gamma B_{2}$ the result is:

$$
\begin{aligned}
& \sqrt{2} R\left(\omega, \vec{r}, B_{1}\right)\left[\cos \omega t \sin \left(\gamma-\frac{\pi}{4}\right)+\sin \omega t \cos \left(\gamma-\frac{\pi}{4}\right) B_{1}\right. \\
& \left.+\cos \omega t \cos \left(\gamma-\frac{\pi}{4}\right) B_{2}+\sin \omega t \sin \left(\gamma-\frac{\pi}{4}\right) B_{3}\right]
\end{aligned}
$$

If sprefield is scattered by $\cos \gamma+\sin \gamma B_{3}$ the result is:

$$
R\left(\omega, \vec{r}, B_{1}\right)\left[\cos (\omega t-\gamma)+\sin (\omega t+\gamma) B_{1}+\cos (\omega t-\gamma) B_{2}+\sin (\omega t+\gamma) B_{3}\right]
$$

All these g-qubits are defined for all values of $t$ and $\vec{r}$, in other words the result of Clifford translation by spreon (1.5) instantly spreads through the whole three-dimensions for all values of time.

The resulting state (10) is simultaneously redefined for all values of $t$. We particularly have changing of state backward in time. That is obvious demonstration that the suggested theory allows indefinite event casual order. In that way the very notion of the concept of cause and effect disappears, thus we might not perceive time.

\section{Measurements by Sprefields}

The Hopf fibration, measurement of any observable $C_{0}+C_{1} B_{1}+C_{2} B_{2}+C_{3} B_{3}$ in the current formalism is [3]: 


$$
\begin{aligned}
& C_{0}+C_{1} B_{1}+C_{2} B_{2}+C_{3} B_{3} \stackrel{\alpha+\beta_{1} B_{1}+\beta_{2} B_{2}+\beta_{3} B_{3}}{\longrightarrow} \\
& C_{0}+\left(C_{1}\left[\left(\alpha^{2}+\beta_{1}^{2}\right)-\left(\beta_{2}^{2}+\beta_{3}^{2}\right)\right]+2 C_{2}\left(\beta_{1} \beta_{2}-\alpha \beta_{3}\right)+2 C_{3}\left(\alpha \beta_{2}+\beta_{1} \beta_{3}\right)\right) B_{1} \\
& +\left(2 C_{1}\left(\alpha \beta_{3}+\beta_{1} \beta_{2}\right)+C_{2}\left[\left(\alpha^{2}+\beta_{2}^{2}\right)-\left(\beta_{1}^{2}+\beta_{3}^{2}\right)\right]+2 C_{3}\left(\beta_{2} \beta_{3}-\alpha \beta_{1}\right)\right) B_{2} \\
& +\left(2 C_{1}\left(\beta_{1} \beta_{3}-\alpha \beta_{2}\right)+2 C_{2}\left(\alpha \beta_{1}+\beta_{2} \beta_{3}\right)+C_{3}\left[\left(\alpha^{2}+\beta_{3}^{2}\right)-\left(\beta_{1}^{2}+\beta_{2}^{2}\right)\right]\right) B_{3}
\end{aligned}
$$

Apply this formula for measurement by spreon (1.5):

$$
2 \cos \omega\left(\left[\left(I_{3} I_{S}\right) \cdot \vec{r}\right]\right)\left(\frac{1}{\sqrt{2}} \cos \omega t+\frac{1}{\sqrt{2}} I_{S} \sin \omega t+\frac{1}{\sqrt{2}} I_{B_{0}} \cos \omega t+\frac{1}{\sqrt{2}} I_{E_{0}} \sin \omega t\right),
$$

that is use:

$$
\begin{gathered}
B_{1}=I_{S}, \quad B_{2}=I_{B_{0}}, \quad B_{3}=I_{E_{0}} \\
\alpha=2 \cos \omega\left[\left(I_{3} I_{S}\right) \cdot \vec{r}\right] \frac{1}{\sqrt{2}} \cos \omega t ; \quad \beta_{1}=2 \cos \omega\left[\left(I_{3} I_{S}\right) \cdot \vec{r}\right] \frac{1}{\sqrt{2}} \sin \omega t \\
\beta_{2}=2 \cos \omega\left[\left(I_{3} I_{S}\right) \cdot \vec{r}\right] \frac{1}{\sqrt{2}} \cos \omega t ; \quad \beta_{3}=2 \cos \omega\left[\left(I_{3} I_{S}\right) \cdot \vec{r}\right] \frac{1}{\sqrt{2}} \sin \omega t
\end{gathered}
$$

The result of measurement is:

$$
\begin{aligned}
& O\left(C_{0}, C_{1}, C_{2}, C_{3}, I_{S}, I_{B_{0}}, I_{E_{0}}, \omega, t, \vec{r}\right) \\
& =4\left(\cos \omega\left[\left(I_{3} I_{S}\right) \cdot \vec{r}\right]\right)^{2}\left[C_{0}+C_{3} I_{S}+\left(C_{1} \sin 2 \omega t+C_{2} \cos 2 \omega t\right) I_{B_{0}}\right. \\
& \left.+\left(C_{2} \sin 2 \omega t-C_{1} \cos 2 \omega t\right) I_{E_{0}}\right]
\end{aligned}
$$

Geometrically, this result means that the observable bivector plane rotates by $\frac{\pi}{2}$ around vector $e_{2}$, such that the $C_{3}$ component becomes lying in plane $I_{S}$. Two other components lying in planes orthogonal to $I_{S}$ rotate around normal to $I_{S}$ with angular velocity $2 \omega t$. Both scalar and bivector parts get scalar factor $4\left(\cos \omega\left[\left(I_{3} I_{S}\right) \cdot \vec{r}\right]\right)^{2}$.

Formula (3.2) shows that only component of the result of measurement lying in plane $I_{S}$ does not depend on the value of time parameter.

We know that any two observables can be connected through Clifford translation. If we are concerned only in the $I_{S}$ component of the result of measurement then with placing another observable value $C_{3}^{\text {new }}$ in (3.1) the latter can be written, in assumption that the $I_{S}$ old observable component is not zero, as:

$$
4 \operatorname{sign}\left(C_{3}\right) \operatorname{sign}\left(C_{3}^{\text {new }}\right)\left(\cos \omega\left[\left(I_{3} I_{S}\right) \cdot \vec{r} \sqrt{\left|\frac{C_{3}^{\text {new }}}{C_{3}}\right|}\right]\right)^{2}\left[\cdots+C_{3} I_{S}+\cdots\right]
$$

Thus, all the $I_{S}$ components of any observable do simultaneously exist whilst we only made measurement of one observable. All the other observables values are calculated at values $\vec{r} \sqrt{\left|\frac{C_{3}^{\text {new }}}{C_{3}}\right|}$.

Consider more complicated way to get component of the result of measurement not depending on time parameter. 
Assume the spreon is scattered by some state $\cos \gamma+\sin \gamma\left(\gamma_{1} I_{S}+\gamma_{2} I_{B_{0}}+\gamma_{3} I_{E_{0}}\right)$ before the measurement. The result of the measurement in general case is a bit tedious. Let us take as the first example the bivector components with $\gamma_{2}=1, \gamma_{1}=\gamma_{3}=0$. In that case the result of measurement, from (3.1), of $C_{0}+C_{1} I_{S}+C_{2} I_{B_{0}}+C_{3} I_{E_{0}}$ can be calculated as its measurement by $\cos \gamma+\sin \gamma I_{B_{0}}$ :

$$
\begin{aligned}
& C_{0}+C_{1} I_{S}+C_{2} I_{B_{0}}+C_{3} I_{E_{0}} \stackrel{\cos \gamma+\sin \gamma I_{B_{0}}}{\longrightarrow} \\
& C_{0}+\left(C_{1} \cos 2 \gamma+C_{3} \sin 2 \gamma\right) I_{S}+C_{2} I_{B_{0}}+\left(C_{3} \cos 2 \gamma-C_{1} \sin 2 \gamma\right) I_{E_{0}}
\end{aligned}
$$

followed by measurement by

$$
2 \cos \omega\left(\left[\left(I_{3} I_{S}\right) \cdot \vec{r}\right]\right)\left(\frac{1}{\sqrt{2}} \cos \omega t+\frac{1}{\sqrt{2}} I_{S} \sin \omega t+\frac{1}{\sqrt{2}} I_{B_{0}} \cos \omega t+\frac{1}{\sqrt{2}} I_{E_{0}} \sin \omega t\right)
$$

that gives, using (3.2):

$$
\begin{aligned}
& 4\left(\cos \omega\left[\left(I_{3} I_{S}\right) \cdot \vec{r}\right]\right)^{2}\left[C_{0}+\left(C_{3} \cos 2 \gamma-C_{1} \sin 2 \gamma\right)\left(\cos 2 \gamma I_{S}-\sin 2 \gamma I_{E_{0}}\right)\right. \\
& +\left(\left(C_{1} \cos 2 \gamma+C_{3} \sin 2 \gamma\right) \sin 2 \omega t+C_{2} \cos 2 \omega t\right) I_{B_{0}} \\
& \left.+\left(C_{2} \sin 2 \omega t-\left(C_{1} \cos 2 \gamma+C_{3} \sin 2 \gamma\right) \cos 2 \omega t\right)\left(\cos 2 \gamma I_{E_{0}}+\sin 2 \gamma I_{S}\right)\right]
\end{aligned}
$$

If we take new orthonormal bivector basis:

$$
\left\{\cos 2 \gamma I_{S}-\sin 2 \gamma I_{E_{0}}, \sin 2 \gamma I_{S}+\cos 2 \gamma I_{S}, I_{B_{0}}\right\} \equiv\left\{I_{1 \gamma}, I_{2 \gamma}, I_{B_{0}}\right\}
$$

the result of measurement reads:

$$
\begin{aligned}
& 4\left(\cos \omega\left[\left(I_{3} I_{S}\right) \cdot \vec{r}\right]\right)^{2}\left[C_{0}+\left(C_{3} \cos 2 \gamma-C_{1} \sin 2 \gamma\right) I_{1 \gamma}\right. \\
& +\left(C_{2} \cos 2 \omega t+\left(C_{1} \cos 2 \gamma+C_{3} \sin 2 \gamma\right) \sin 2 \omega t\right) I_{B_{0}} \\
& \left.+\left(C_{2} \sin 2 \omega t-\left(C_{1} \cos 2 \gamma+C_{3} \sin 2 \gamma\right) \cos 2 \omega t\right) I_{2 \gamma}\right]
\end{aligned}
$$

that has constant value in plane $I_{1 \gamma}$ plus rotation in planes $I_{B_{0}}$ and $I_{2 \gamma}$ with angular velocity $2 \omega$.

In that way we particularly get $\vec{r}$-dependent variety of constant components of the results of measurements:

$$
4\left(\cos \omega\left[\left(I_{3} I_{S}\right) \cdot \vec{r}\right]\right)^{2}\left[C_{0}+\left(C_{3} \cos 2 \gamma-C_{1} \sin 2 \gamma\right) I_{1 \gamma}\right]
$$

Similar results are for other cases of scattering state: $\gamma_{1}=1, \gamma_{2}=\gamma_{3}=0$ and $\gamma_{3}=1, \gamma_{1}=\gamma_{2}=0$.

\section{Conclusions}

All measured observable values are instantly spread through the whole set of three-dimension points and time parameter values. If the measuring results represent a function value, the values are available altogether, not through evaluating one by one.

The current approach transcends qubit entangled computational schemes since the latter have tough problems of creating large sets of communicating qubits. All the efforts today in building "quantum" computers are in implementa- 
tion of qubits (in various physically possible variants) effectively talking to each other, thus emulating entanglement.

In the current scheme any observable can be placed into continuum of the $(t, \vec{r})$ dependent values of the sprefield. All other observables' measurement results are particularly connected by Clifford translations thus giving any number of values $O\left(C_{0}, C_{1}, C_{2}, C_{3}, I_{S}, I_{B_{0}}, I_{E_{0}}, \omega, t, \vec{r}\right)$, spread over three-dimensions and at all instants of time not generally following cause/effect ordering.

\section{Conflicts of Interest}

The author declares no conflicts of interest regarding the publication of this paper.

\section{References}

[1] Hestenes, D. (1999) New Foundations for Classical Mechanics. Kluwer Academic Publishers, Dordrecht/Boston/London.

[2] Doran, C. and Lasenby, A. (2010) Geometric Algebra for Physicists. Cambridge University Press, Cambridge.

[3] Soiguine, A. (2020) The Geometric Algebra Lift of Qubits and Beyond. Academic Publishing, Lambert.

[4] Soiguine, A.M. (1996) Complex Conjugation-Relative to What? In: Rafał, A., Josep, M.P.L., Eds., Clifford Algebras with Numeric and Symbolic Computations, Boston, Birkhauser, 284-294. https://doi.org/10.1007/978-1-4615-8157-4_19

[5] Soiguine, A. (2018) State/Observable Interactions Using Basic Geometric Algebra Solutions of the Maxwell Equation. arXiv:1807.08603.

[6] Soiguine, A. (2020) Scattering of Geometric Algebra Wave Functions and Collaps in Measurements. Journal of Applied Mathematics and Physics, 8, 1838-1844. https://doi.org/10.4236/jamp.2020.89138 